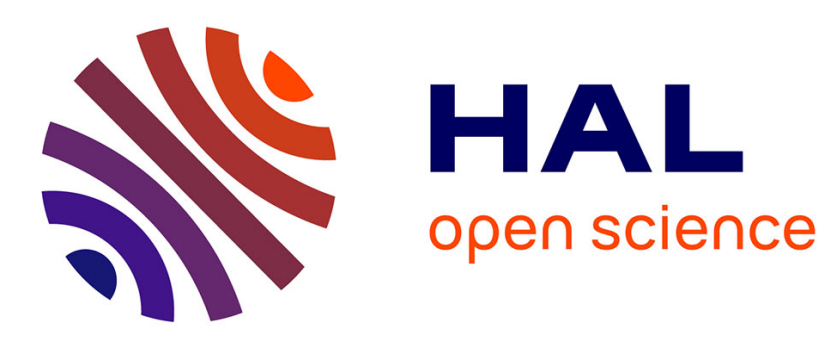

\title{
Designing an Accessible and Engaging Email Application for Aging in Place
}

Loïc Caroux, Charles Consel, Hélène Sauzéon, Lucile Dupuy

\section{To cite this version:}

Loïc Caroux, Charles Consel, Hélène Sauzéon, Lucile Dupuy. Designing an Accessible and Engaging Email Application for Aging in Place. UIC 2017 - IEEE International Conference on Ubiquitous Intelligence and Computing, Aug 2017, San Francisco, United States. pp.8. hal-01556783

\section{HAL Id: hal-01556783 \\ https://hal.inria.fr/hal-01556783}

Submitted on 5 Jul 2017

HAL is a multi-disciplinary open access archive for the deposit and dissemination of scientific research documents, whether they are published or not. The documents may come from teaching and research institutions in France or abroad, or from public or private research centers.
L'archive ouverte pluridisciplinaire HAL, est destinée au dépôt et à la diffusion de documents scientifiques de niveau recherche, publiés ou non, émanant des établissements d'enseignement et de recherche français ou étrangers, des laboratoires publics ou privés. 


\section{Designing an Accessible and Engaging Email Application for Aging in Place}

\author{
Loïc Caroux \\ University of Toulouse \\ Toulouse, France \\ loic.caroux@univ-tlse2.fr
}

\author{
Charles Consel \\ Bordeaux INP / Inria \\ Bordeaux, France \\ charles.consel@inria.fr
}

\author{
Hélène Sauzéon \\ University of Bordeaux / Inria \\ Bordeaux, France \\ helene.sauzeon@inria.fr
}

\author{
Lucile Dupuy \\ Inria \\ Bordeaux, France \\ lucile.dupuy@inria.fr
}

\begin{abstract}
Supporting independent everyday functioning of older adults is a major challenge for aging in place. In particular, communication and social activities need support to prevent social isolation, cognitive and psychosocial well-being decline, and a risk of depression.

This paper focuses on how technology can bring social support to isolated older-old adults (over 75 years old) and allow them to communicate with members of their social network. We present the design of an accessible and engaging email application dedicated to this population. We propose design principles based on the older adults' specificities and then use these principles to develop a tablet-based email application.

We conducted a field study to evaluate our email application during 9 months. We equipped 13 community-dwelling oldolder adults with a touchscreen tablet and our application at their home (compared to 13 control counterparts). This field study validates our design principles as shown by the effectiveness and efficiency gained by the participants in using our application. Moreover, we reveal the influence of health indicators in the usage behaviors and the long-term use of our application.
\end{abstract}

\section{INTRODUCTION}

Assistive computing is a promising research field for aging in place. Assistive technology can support independent living for older adults, in particular by assisting them in most of their activities of daily living. Supporting independent everyday functioning of this population may prevent or reduce cognitive or sensory-motor decline. The literature on aging usually proposes three categories of activities of daily living for which older adults wish and need to be assisted with technology: daily activities (e.g., medication reminder, meal preparation monitoring), personal safety and home security (e.g., stove monitoring, detecting of unusual situations), and communication and social activities (e.g., agenda, remote communication) [1], [2].

Regarding communication and social activities, older adults, and especially old-older adults (over 75 years of age), need support to prevent social isolation and a risk of depression. As individuals age, they are likely to loose friends, family members, and above all their spouse. Also, aging means that physical and cognitive resources of the person may decline. Consequently, communication skills decrease while psycho-affective difficulties appear [3]. Social isolation is a societal challenge because it impacts not only older adults but also their caregivers who are acting as human support for the performance of everyday activities. These supporting activities may become a burden depending on the older adult's difficulties.

In this paper, we focus on how technology can bring social support to isolated old-older adults to communicate with members of their social network, including family members, peers, and caregivers. This kind of technology aims to support the cognitive and psychosocial well-being of older adults for aging in place.

Current assistive technologies allow users to communicate whenever they want, however they want, with whoever they want. However, there is a critical gap between the promises of these technologies and the actual needs and skills of older adults. To close this gap, the technology must be accessible and engaging. It should be designed so that the demands of the technology and the underlying tasks are amenable to the needs and skills of the person [4].

Nowadays, email-based communication is popular among younger adults. This mode of communication has the advantage of being indirect and asynchronous. In fact, more direct modes of communication, such as video/voice calls, would be better suited to address social isolation and the decline of communication skills [5]. However, these more direct modes of communication are inappropriate in the context of aging because older adults mainly target their descendants (children and grand-children) for their social interactions [3], while these individuals are often busy with their professional and family activities. Usually, there is a mismatch between the schedule of older adults and the one of their descendants, preventing mutual acceptance of communication via direct and synchronous modes. Therefore, an indirect, asynchronous mode of communication, such as email, is an ideal solution for both parties.

The problem of existing email applications is that basic operations (reading, writing, receiving, sending) are not easily accessible, neither engaging for older adults with low or no computer skills, or with pronounced cognitive or physical losses [6]. To remedy this situation, we need to elaborate principles to design accessible and engaging applications, based on needs and skills of older adults.

This paper presents the design of an email application that is accessible and engaging for older adults, support- 
ing independent aging in place. First, we examine the specificities of older adults and specify the email activity. Second, we propose design principles on the basis of these specifications. Third, we describe the features of our email application, whose design relies on these principles. Finally, we present a field study with 13 older-old adults using this application during 9 months, compared to 13 control counterparts. We demonstrate the impact of our application on their communication behaviors and several well-being indicators (cognitive functioning and self-perceived health).

\section{SPECIFICATION}

To design our new email application, we first examine the specificities of the target users and the specification of the target domain of activity. In the present case, we establish the characteristics of older adults and the email activities.

Regarding older adults, two sets of characteristics must be considered from the literature on aging. First, cognitive and sensory-motor resources may decline when a person ages. Second, several barriers due to aging exist before technologies are accepted and, in a longer perspective, adopted.

\section{A. Declining cognitive and sensory-motor resources}

While many individuals experience healthy aging, without significant impairments, a number of sensory, motor and cognitive declines can occur with aging. Sensory resources mostly decline through a decrease of visual acuity and contrast sensitivity. Also, there may be a decrease of hearing of high-frequency sounds and background sounds inhibiting. Regarding motor skills, movements made by older adults tend to be slower and less precise than those made by younger adults. Furthermore, cognitive abilities, primarily related to memory, planning and executing tasks, are well documented as major underpinnings of the functional status of older adults with and without cognitive impairment. Fisk et al. provide a comprehensive list of typical resources declining with age, and explain how they decline [7]. Moreover, it has been shown that when growing old, intra- and inter-variability is accentuated; this situation has to be taken into account when designing technologies [8].

\section{B. Barriers to technology acceptance}

Older adults do accept and use technology, and especially communication technology such as telephone. However, several barriers to technology have been highlighted for the population of older users [9], [10]. In the Senior Technology Acceptance Model (STAM), Chen \& Chan [11] showed that health and physical conditions, as well as perceived technical skills for computer-based tools, have an impact on both technology acceptance and perceived ease of use and usefulness of a technology. Therefore, acceptance of communication tools (email, video/voice calls, etc.) can be compromised when their design are not accessible and engaging for older adults.

\section{Email-based communication activities}

When examining the email activities to be performed by a user, we identified email tasks that revolve around two key notions: messages and contacts. More precisely, messages have to be composed (e.g., create a new message, reply to a message), viewed (e.g., received messages), and managed (e.g., removed). Additionally, there needs to be support to manage the user's contacts.

\section{DESIGN PRINCIPLES}

In this section, we propose several design principles that meet the requirements of older adults to perform the tasks involved in email activities. These principles contribute to achieve our goal to make the demands of the technology and the tasks amenable to the various and multiple needs and skills of older adults.

P1: Accessible visual rendering: Existing principles of visual rendering have to be applied. But, since sensory-motor resources of older adults are specific, standards dedicated to interface design for older adults should be followed (e.g., ISO/TR 22411:2008 [12]). For example, text fonts should be high enough to be easily readable. Colors (including hue and luminance) should be chosen to obtain high-contrast ratios between text and background. We also leverage the comprehensive list of principles to design displays for older adults provided by Pak and McLaughlin [13].

P2: Multi-coding of displayed information: Following the principles of Pak and McLaughlin [13], the information contained in received messages should be available in different codes whenever possible. For example, the users should view a message in a way that is the most compatible with their sensory capabilities. In particular, the tool should allow the users to read a message or to listen it via text-to-speech.

P3: Multi-modality to compose a message: Different strategies for the composition of a message should also be proposed. For example, the user should compose a message according to the motor capabilities of their upper limb. In particular, the message text should either be typed on a keyboard or voice recorded.

P4: Over-simplifying interactions with technology: To account for the declining cognitive resources of older adults, not only should the use of technology be easy, but it should also be as much simplified as possible. Technology should be designed in such a way that the users can only focus on the main task: the message. The aim of this principle is to limit the required amount of cognitive resources to interact with the technology. The number of navigation steps to be managed to achieve an action should be kept to a minimum [7]. Distractive parameters or non-essential actions should be avoided [14].

P5: Consistent design: In compliance to the previous principle, interactions and interfaces should be consistent throughout the application. Again, this principle aims to limit the required amount of cognitive resources to process 
the displayed information. Also, new situations can unnecessarily require cognitive resources. The users will be more effective if a given task and its underlying actions and parameters are as invariant as possible. Furthermore, this principle contributes to prevent interaction errors.

P6: Leveraging people's knowledge of traditional technology: Older adults are users of existing communication technology. To break barriers of acceptance, the new technology should be designed using standards and affordances from already-accepted technologies. In particular, design should leverage knowledge of traditional communication technology, such as telephone or even surface mailing.

P7: Automation of actions: Automating actions can also contribute to breaking barriers of acceptance. Actions that are not essential to the email tasks should be performed by the application, not by the user. These automated actions should not compromise the standard usage of the communication tool. Instead, they should skip over details whose omission is acceptable to message recipients, aware of the technology skills of the user and/or their desire to minimize interactions with technology. Also, the automation of actions can reduce the required number of steps to perform a task, which may prevent users from getting lost or disoriented during the task performance.

P8: Preventing solicitation from unintended contacts: Acceptance of technology can be compromised if older adults are solicited by messages from unintended or unknown persons or institutions. The application should prevent these situations, constraining the behavior of the technology within a well-defined, customized scope to match the expectations and skills of the user.

Even if this paper proposes principles to design an email application for older adults, some of these principles could be used for other kinds of communication tools, from the most traditional ones, such as a telephone, to the most innovative ones, such as a Web browser.

\section{OUR APPLICATION}

We designed and developed a fully functional tablet-based email application for older adults, following the principles presented above. This section describes the salient features of this application and illustrate them with screenshots of the actual interfaces of the application. Each of the design principles was addressed with one or more features in the application. The notation (P1) refers to the design principle 'P1' introduced earlier. Note that one feature may correspond to more than one design principle.

Our email application is dedicated to touchscreen tablets, which are known to be accessible devices and allow natural interaction for older adults, in comparison to personal computers [15]. However, most of our proposed features are relevant for other kinds of computer devices (e.g., smartphone, personal computer).
The visual interface rendering is accessible for older adults (P1): We use standards dedicated to interface design for older adults and map them to touchscreen tablets. In particular, text fonts and contrast ratios were carefully selected. An example of interface rendering of our application is shown in Figure 1

Contacts are displayed with their name and their picture and easily managed $(\boldsymbol{P 2}, \boldsymbol{P 4})$ : Information is multi-coded when possible. A contact is represented by their name, but also by their photo or any picture of the user's choice (Figure 11). Contacts can be reorganized simply with a drag gesture; a scroll gesture can show hidden contacts (these gestures have shown to be easily managed by older users [16]).

Received messages can be read or listened to (P2): Users have the choice to read a message or to listen to it. In the latter case, a speech synthesizer reads out loud the message (Figure 2).

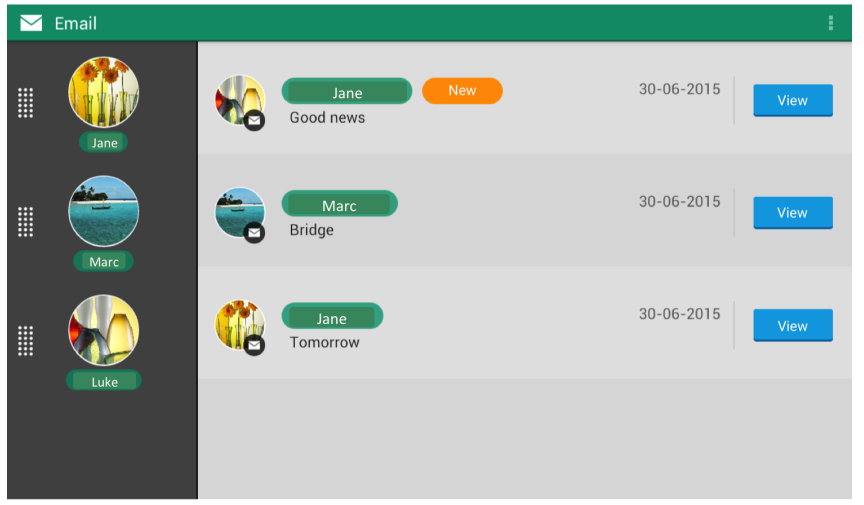

Figure 1: Main screen of the email application. The left pane contains the contacts selected by the user and represented by their name and picture. The user taps on a contact picture or name to compose a new message. The right pane contains messages in the inbox. The user taps on 'View' to view the message.

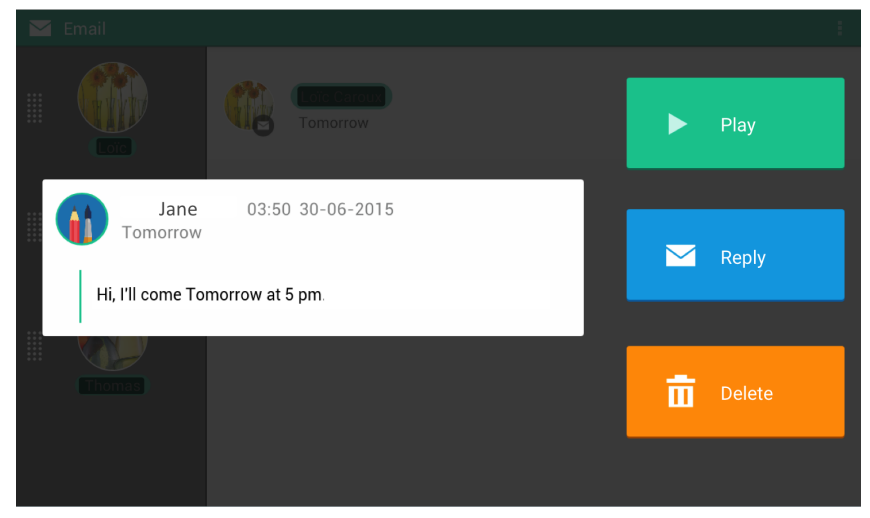

Figure 2: Viewing a message. The message is displayed in the white frame. Only three actions are possible at this step. The user can listen to the message via the voice synthesizer, reply to it, or delete it. If the message was voice-recorded by the sender, this is indicated by a message displayed in the white frame; tapping on 'Play' then plays the audio file. 
A message can be typed or voice recorded $(\mathbf{P 3}, \mathbf{P 6})$ : Users have the choice to create a new message or to reply to a message using text or voice recording (Figure 3). The possibility to send a voice-recorded message leverages the traditional telephone mode of communication. When the message is voice-recorded, it is sent as an attached file to an email message. This file is recorded in a standard format so that the receiver can easily play it with a standard email client. This kind of interaction mimics a traditional answering machine. The interface to write a message is displayed in Figure 4 The interface to record a message is displayed in Figure 5.

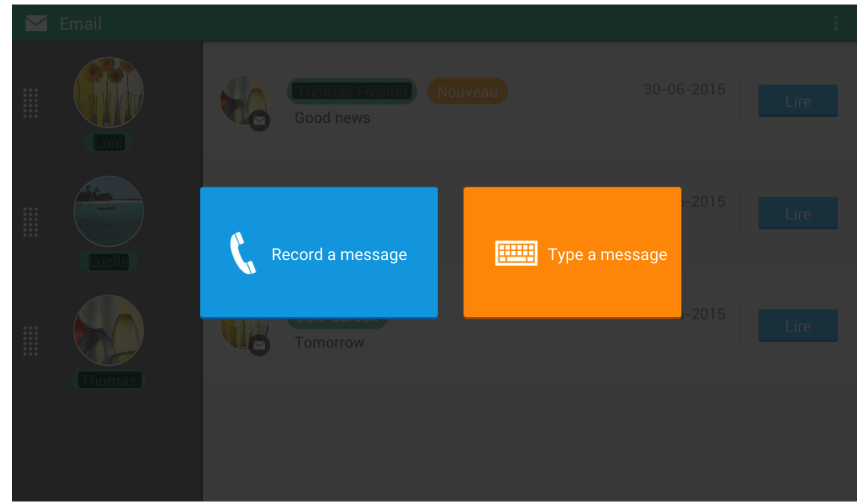

Figure 3: A choice to record or to type a message. This interface is displayed when the user taps on a contact icon or on 'Reply' to a message.

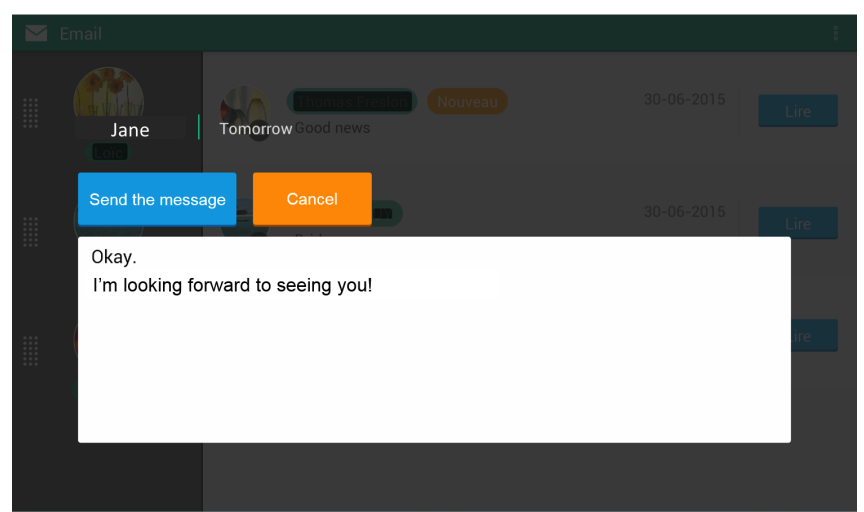

Figure 4: Writing a message. The message can be typed with the virtual keyboard of the tablet. When the message is composed, only two actions are possible: sending it or cancelling it.

The application is always displayed in a full-screen mode (P4): The design of the application follows a singletask paradigm. When users are involved in a task (e.g., composing a message, reading a message), they cannot be disturbed by another task or they cannot engage in another task.

The steps for a given task are sequential and numbered $(\boldsymbol{P 4}, \boldsymbol{P 5}, \boldsymbol{P 6})$ : In compliance with the single-task paradigm, users are guided from the start to the end of a task, as

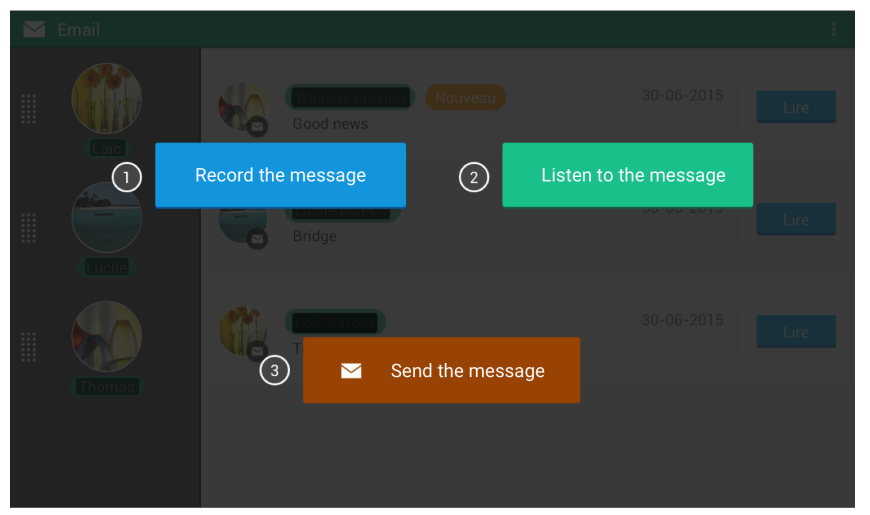

Figure 5: Recording a message. Only three actions are possible here. The interface makes explicit the order in which they have to be performed. First, recording the message, second, listening to the recorded message, and third, sending it. However, the action of recording or listening can be done an unlimited number of times before sending the message.

illustrated by the recording of a message displayed in Figure 5

Non-essential fields during message composition are hidden or automatically filled (P4, P7): When users want to compose and send a message, they choose a person from their contact list and then compose the message before sending it. Non-essential message fields, such as the email address of the receiver, are hidden from users. Other fields are filled automatically (e.g., subject) or not offered as parameters (e.g., carbon-copy).

Dedicated buttons are labeled with standard terms of communication technology (P6): Each action is accessible via a button that is labeled with a common term, leveraging older adults' traditional technology skills. This is illustrated in Figure 5, where the task of recording a message uses terms and icons from traditional telephone answering machines.

Messages can only be received from or sent to predefined contacts (P8): Only messages from contacts included in the user's contact list are received and can be viewed. Similarly, users can only send a message to someone from their contact list (Figure 11). Note that not only does our application include a feature to introduce contacts, but it also allows contacts to be added remotely by authorized caregivers, lifting this burden off the users.

\section{Evaluation METHOD}

We designed a field study to evaluate our application. We equipped 13 community-dwelling old-older adults with our application installed on a touchscreen tablet at their home. The aim of this study was to evaluate the usability of the application by older adults, over a period of 9 months. Furthermore, this study was designed to assess whether, and to what extent, such a tool supports the cognitive and psychosocial well-being of older adults for aging in place. To this end, we included a control group to compare equipped $v s$. non-equipped older adults. 
At the beginning of the 9-month period, the participants received a short training by the experimenter. Then, they were asked to use the application, installed on a tablet, in any way they wanted during this period.

During the experiment, measurements were made at three different stages: just after deploying our tablet-based email application $(t 0)$, after six months of using it (intermediate evaluation, $t 6$ ), and after 9 months of use (final evaluation, $t 9)$. The objective of this evaluation method is to evaluate (1) the usability of the application and its learnability across time and (2) the benefits in terms of cognitive functioning and psychosocial health for the older adults.

\section{A. Participants}

We recruited 26 community-dwelling older adults, in collaboration with a public home care service for older adults. All participants lived alone in their own home. Our participants were recruited according to specific exclusion criteria: dependency syndrom, neurological or musculoskeletal disease or systemic disorder. The main inclusion criterion was cognitive integrity with an MMSE (Mini Mental State Examination [17]) greater than 24. All participants provided a written consent prior to participating to our study.

Our participants were split into two groups, matched with age, gender, MMSE and education years (Table (I). One group used our tablet-based email application. The other group formed a control group. Our goal was to observe the health-related benefits of using our communication application, by comparing measures between equipped and non-equipped participants.

\begin{tabular}{lccc} 
& Table I: Participants profiles & \\
\hline & $\begin{array}{c}\text { Equipped } \\
\text { group } \\
\text { Mean }(S D)\end{array}$ & $\begin{array}{c}\text { Control } \\
\text { group } \\
\text { Mean }(S D)\end{array}$ & $\begin{array}{c}\text { Group } \\
\text { comparison }\end{array}$ \\
\hline Age & $79.00(1.46)$ & $82.23(1.52)$ & $\mathrm{p}>.100$ \\
Gender & 4 males & 2 males & $\mathrm{p}>.300$ \\
MMSE & $27.67(.41)$ & $28.38(.40)$ & $\mathrm{p}>.200$ \\
Education years & $9.61(.55)$ & $9.61(.66)$ & $\mathrm{p}>.900$ \\
\hline & $S D=$ Standard Deviation &
\end{tabular}

\section{B. Data Collected}

Every measurement was made three times: at the beginning of the experiment ( $t 0)$, after six months of usage $(t 6)$, and after nine months, at the end of the experiment $(t 9)$.

1) Timed usage scenario for assessing email application usability: Inspired by the timed-IADL tasks [18], we designed a usage scenario to assess the performance of a user performing a task with our application within an allocated time. The scenario focuses on the activity of sending an email message rather than receiving one. Indeed, the former task involves more action steps, and thus aims to further test usage behaviors of participants. The scenario was defined as follows.

- Task condition: The user holds the tablet that displays the main menu.
- Instructions to the user: "Could you send me an email? You do not have to write (or say) anything, it is just to see how you manage the activity of sending email."

- Time ends when: The user taps on "send the message" button.

- Allocated time: 1 minute.

This test provides two usability measures. First, an effectiveness measure is done. It ranges over a scale of 3 ; the score depends on the number of errors made by the user, and whether the task was performed within the allocated time. A score of 3 denotes a task performed without errors and within the allocated time. A score of 2 corresponds to a task performed with errors but within the allocated time. A score of 1 is when the user did not complete the task within the allocated time. Second, an efficiency measure is also collected thanks to the recording of the accurate time in seconds to perform the usage task.

2) Cognitive and health indicators: Cognitive functioning. Several tests, well-known to be sensitive to the aging effect [19], were used to assess the following cognitive aspects.

- The global cognitive functioning: measured with the Dementia Rating Scale 2 (DRS-2) [20].

- The executive functioning: measured with the Frontal Assessment Battery (FAB) [21], the Trail Making Test (TMT) [22] and the Victoria Stroop Test [23]. We used the ratio $\left(T M T_{B}-T M T_{A}\right) / T M T_{A}$ for time in the TMT, and the two interference scores Word/Dot and Interference/Dot for time in the Victoria Stroop.

- The memory functioning: measured with the Benton Visual Retention Test [24] and the Five Words Test [25].

- The socio-cognitive functioning: measured with the "Reading the Mind in the Eyes" test [26].

We computed Z-scores for all these tests, and made the sum to obtain an indicator of cognitive functioning, where higher values indicate greater cognitive functioning.

Self-perceived health. For this indicator, we used the following tests.

- The Short Form 36 of Health Survey (SF-36) [27], inducing two sub-scores: physical and mental perceived health, higher scores indicating better self-perceived health.

- The 28-item General Health Questionnaire (GHQ28) [28], scored over 96, higher scores indicating more general health-related problems.

We computed the Z-scores of these two tests, and made the sum to generate an indicator of perceived health, higher scores indicating better perceived health.

Expected results of the experimental study are that our application (1) is usable and engaging for older adults (i.e., easy to use and learn), and (2) can support aging in place by 
contributing to prevent the loss of cognitive functioning and self-perceived health (in particular the mental dimension).

\section{RESULTS}

To achieve our research objectives, we performed the following statistical analyses: (1) univariate repeated ANOVA for time (i.e., t0 vs. t6 vs. $t 9$ ) to reveal changes across time in terms of effectiveness, efficiency and (2) mixed ANOVA with time and group of subjects (i.e., equipped $v s$. control) as independent factors and with cognitive and health indicators as dependent factors, to highlight the benefits brought by the use of our application.

\section{A. Usability measures across time}

Figure 6 plots the two usability scores (effectiveness and efficiency) from $t 0$ to $t 9$.

As illustrated, the effectiveness score for performing the timed-usage scenario increases across time $(\mathrm{F}(2,24)=7.70$; $\mathrm{p}=.003)$. Conjointly, a strong enhancement of efficiency score is observed (i.e., decrease of performance time; $\mathrm{F}(2,24)$ $=7.95 ; \mathrm{p}=.002)$. This means that both effectiveness and efficiency dimensions of the usability of our application enhance across time. In other words, older users learned accurately how to use our application, becoming agile with it.
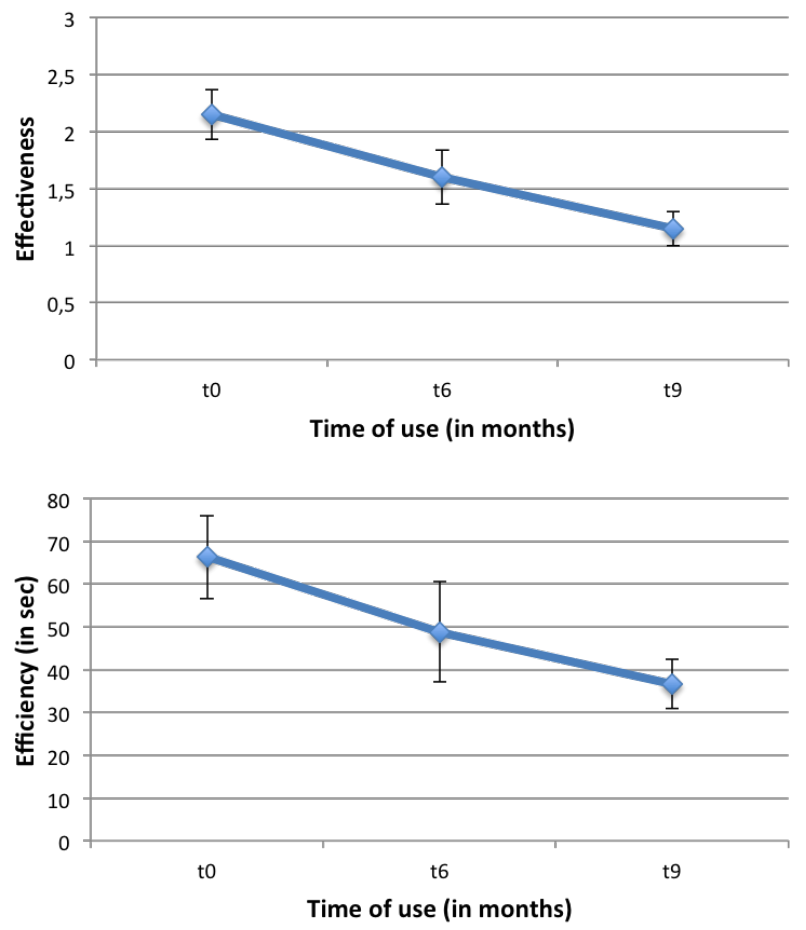

Figure 6: Usability measures across time (t0 vs. t6 vs. t9) with (on the top) effectiveness and (on the bottom) efficiency score (in sec.). Error bars represent standard errors.

\section{B. Benefits of using our email application}

Table II summarizes the means and standard deviation in terms of cognitive functioning and perceived health for our two groups of participants. For the cognitive functioning indicator, significant Time $\times$ Group interaction effect is obtained $(\mathrm{F}(1,23)=7.12 ; \mathrm{p}=.004)$, given that the control group exhibits a decrease of cognitive functioning across time, while the equipped group exhibits an increase of the cognitive functionning indicator. Note that no significant effect is reported for the two other health indicators (i.e., physical functioning and self-perceived health). Overall, the use of our email application improves only the cognitive functioning of the users. Also, there is probably not enough cognitive benefits to induce a positive change on the general self-perceived health indicator. Indeed, this indicator is multidetermined, notably by physical functionning that obviously is not changed by the use of our email application.

Table II: Means and standard deviation of cognitive and health indicators across time for our two groups of participants

\begin{tabular}{clcc}
\hline & & $\begin{array}{c}\text { Equipped } \\
\text { group } \\
\text { Mean }(S D)\end{array}$ & $\begin{array}{c}\text { Control } \\
\text { group } \\
\text { Mean }(S D)\end{array}$ \\
\hline$t 0$ & cognitive funct. & $-.01(1.00)$ & $.03(1.15)$ \\
& perceived health & $.06(1.75)$ & $-.33(1.73)$ \\
\hline$t 6$ & cognitive funct. & $-.06(.13)$ & $.07(.38)$ \\
& perceived health & $.08(1.30)$ & $-.33(2.26)$ \\
\hline$t 9$ & cognitive funct. & $\mathbf{. 0 7}(.16)$ & $\mathbf{- . 1 4}(.32)$ \\
& perceived health & $.20(1.27)$ & $-.23(2.25)$ \\
\hline \multicolumn{3}{c}{ SD=Standard Deviation. } \\
\multicolumn{3}{c}{ Salues in boldface indicate significative differences }
\end{tabular}

\section{DISCUSSION}

The purpose of the study was to assess design principles to develop an email application supporting social participation of older adults. The relevance of these design principles leverages the literature on aging and are now also underpinned by a field study. We report two new results.

First, usability measures demonstrate that the email application is quickly mastered by the older users. Indeed, they are accurate as well as diligent to perform the more complex scenario of email usage (message sending task). Moreover, the usability (i.e., effectiveness and efficiency) measures improve over time (from $t 0$ to $t 9$ ), suggesting a high learnability of our application.

Second, regarding well-being impact, our results highlight the benefits brought by the use of our email application, in terms of cognitive and health indicators. Indeed, we observed an improvement over time of cognitive functioning for the equipped group, compared to the control group. This result enhances the positive impact of using our email application, and fits with previous findings stressing the impact of the social network on cognitive abilities [29]. However, no benefits was found on the perceived health conditions compared to our control group. Logically, it can be explained by the fact that a communication application 
has not enough impact on everyday life to improve such dimensions.

Limitations Despite our encouraging results, several limitations need to be mentioned. First, results could be more statistically robust with a larger sample of older adults. Second, the experimental design could have included an experimental condition with an on-the-shelf email application. Indeed, such a condition could magnify the accessibility properties of our email application in demonstrating that an on-the-shelf email tool exhibits poorer performance than our email tool, in terms of usability, as well as actual usage by our older participants. Third, a participatory design approach, involving older adults [30], [31], including for instance focus groups before the field experiment, could have allowed to discover that augmentative communication functionality (i.e., the audio mode) should have been more customizable according to users' learning abilities.

Overall, the present results highlight the relevance of our design specifications, and provide insights regarding factors influencing the intention to use a communication application, and in the longer term, communication technology adoption.

\section{CONCLUSION}

Supporting communication and social activities is a major challenge for aging in place. Specific applications have to be designed to support older adults for remote communication with their family, caregivers and peers. Communication technology is now pervasive and requires their design to be accessible and engaging.

We have proposed design principles that account for the specificities of older adults and have applied these principles to an email application.

We have conducted a field study to evaluate our email application over a period of 9 months. We have equipped the home of 13 community-dwelling old-older adults with a touchscreen tablet and our application, and have recruited non-equipped 13 control counterparts. This field study have validated our design principles as shown by the effectiveness and efficiency gained by the participants in using our application. Furthermore, we have demonstrated the benefits of using our application in terms of cognitive functioning, as compared with the control group.

\section{REFERENCES}

[1] R. M. Baecker, K. Moffatt, and M. Massimi, "Technologies for aging gracefully," Interactions, vol. 19, no. 3, pp. 32-36, 2012.

[2] L. Dupuy, H. Sauzéon, and C. Consel, "Perceived needs for assistive technologies in older adults and their caregivers," in ACM WomENcourage 15', Sep. 2015.

[3] C. Wrzus, M. Hänel, J. Wagner, and F. J. Neyer, "Social network changes and life events across the life span: A metaanalysis." Psychological Bulletin, vol. 139, pp. 53-80, 2013.
[4] S. J. Czaja and S. N. Nair, "Human factors engineering and systems design," in Handbook of Human Factors and Ergonomics, G. Salvendy, Ed. Hoboken, NJ, USA: John Wiley \& Sons, Inc., Mar. 2012, pp. 38-56.

[5] N. Charness, D. C. Parks, and B. A. Sabel, Eds., Communication, technology and aging: opportunities and challenges for the future. New York: Springer Pub. Co, 2001.

[6] N. M. Gell, D. E. Rosenberg, G. Demiris, A. Z. LaCroix, and K. V. Patel, "Patterns of technology use among older adults with and without disabilities," The Gerontologist, vol. 55, pp. 412-421, 2015.

[7] A. D. Fisk, W. A. Rogers, N. Charness, S. J. Czaja, and J. Sharit, Designing for older adults: Principles and creative human factors approaches, 2nd ed., ser. Human factors \& aging series. Boca Raton: CRC Press, 2009.

[8] J. Vines, G. Pritchard, P. Wright, P. Olivier, and K. Brittain, "An age-old problem: Examining the discourses of ageing in hci and strategies for future research," ACM Transactions on Computer-Human Interaction (TOCHI), vol. 22, no. 1, p. 2 , 2015.

[9] S. T. M. Peek, E. J. M. Wouters, J. van Hoof, K. G. Luijkx, H. R. Boeije, and H. J. M. Vrijhoef, "Factors influencing acceptance of technology for aging in place: a systematic review." International journal of medical informatics, vol. 83, no. 4, pp. 235-48, Apr. 2014.

[10] S. J. Czaja, N. Charness, A. D. Fisk, C. Hertzog, S. N. Nair, W. a. Rogers, and J. Sharit, "Factors predicting the use of technology: findings from the center for research and education on aging and technology enhancement (CREATE)." Psychology and aging, vol. 21, no. 2, pp. 333-52, Jun. 2006.

[11] K. Chen and A. H. S. Chan, "Gerontechnology acceptance by elderly Hong Kong Chinese: a senior technology acceptance model (STAM)," Ergonomics, vol. 57, pp. 635-652, 2014.

[12] International Organization for Standardization, Ergonomics data and guidelines for the application of ISO/IEC Guide 71 to products and services to address the needs of older persons and persons with disabilities (ISO/TR 22411:2008), 2008.

[13] R. Pak and A. McLaughlin, Designing displays for older adults, ser. Human factors and aging series. Boca Raton, FL: CRC Press, 2011.

[14] U. Lindenberger, M. Lövdén, M. Schellenbach, S.-C. Li, and A. KrÃijger, "Psychological principles of successful aging technologies: A mini-review," Gerontology, vol. 54, pp. 5968, 2008.

[15] H. Wandke, M. Sengpiel, and M. Sönksen, "Myths about older people's use of information and communication technology," Gerontology, vol. 58, pp. 564-570, 2012.

[16] L. G. Motti, N. Vigouroux, and P. Gorce, "Interaction techniques for older adults using touchscreen devices: a literature review," in Proceedings of the 25th IEME conference francophone on l'Interaction Homme-Machine. ACM, 2013, p. 125 . 
[17] M. F. Folstein, S. E. Folstein, and P. R. McHugh, ""minimental state": a practical method for grading the cognitive state of patients for the clinician," Journal of psychiatric research, vol. 12, no. 3, pp. 189-198, 1975.

[18] C. Owsley, M. Sloane, G. McGwin Jr, and K. Ball, "Timed instrumental activities of daily living tasks: Relationship to cognitive function and everyday performance assessments in older adults," Gerontology, vol. 48, no. 4, pp. 254-265, 2002.

[19] F. I. Craik and T. A. Salthouse, The handbook of aging and cognition. Psychology Press, 2011.

[20] P. J. Jurica, C. L. Leitten, and S. Mattis, DRS-2: Dementia Rating Scale-2, psychological assessment resources eds. ed., Odessa: FI, 2001.

[21] B. Dubois, A. Slachevsky, I. Litvan, and B. Pillon, "The FAB: A frontal assessment battery at bedside," Neurology, vol. 55, pp. 1621-1626, 2000.

[22] R. M. Reitan, "Validity of the trail making test as an indicator of organic brain damage," Perceptual and motor skills, vol. 8, no. 3, pp. 271-276, 1958.

[23] E. Strauss, E. M. Sherman, and O. Spreen, A compendium of neuropsychological tests: Administration, norms, and commentary. Oxford University Press, USA, 1991.

[24] A. B. Sivan, Benton visual retention test. Psychological Corporation San Antonio, TX, 1992.

[25] B. Dubois, F. Touchon, J ans Portet, P.-J. Ousset, B. Vellas, and B. Michel, ""les 5 mots", épreuve simple et sensible pour le diagnostic de la maladie d'alzheimer," Presse Med, vol. 31, pp. 1696-9, 2002.

[26] S. Baron-Cohen, S. Wheelwright, J. Hill, Y. Raste, and I. Plumb, "The "reading the mind in the eyes" test revised version: A study with normal adults, and adults with asperger syndrome or high-functioning autism," Journal of child psychology and psychiatry, vol. 42, no. 2, pp. 241-251, 2001.

[27] J. E. Brazier, R. Harper, N. M. Jones, A. O'Cathain, K. J. Thomas, T. Usherwood, and L. Westlake, "Validating the SF36 health survey questionnaire: new outcome measure for primary care." $B M J$, vol. 305, no. 6846, pp. 160-164, 1992.

[28] D. P. Goldberg and V. F. Hillier, "A scaled version of the General Health Questionnaire," Psychological Medicine, vol. 9, pp. 139-145, 1979.

[29] R. Stoykova, F. Matharan, J.-F. Dartigues, and H. Amieva, "Impact of social network on cognitive performances and age-related cognitive decline across a 20-year follow-up," International Psychogeriatrics, vol. 23, no. 09, pp. 14051412, 2011.

[30] O. Demirbilek and H. Demirkan, "Universal product design involving elderly users: a participatory design model," Applied ergonomics, vol. 35, no. 4, pp. 361-370, 2004.

[31] S. Lindsay, D. Jackson, G. Schofield, and P. Olivier, "Engaging older people using participatory design," in Proceedings of the SIGCHI Conference on Human Factors in Computing Systems. ACM, 2012, pp. 1199-1208. 